\title{
On splittable colorings of graphs and hypergraphs
}

\author{
Zoltán Füredi* \\ DEPARTMENT OF MATHEMATICS, UNIVERSITY OF ILLINOIS, \\ URBANA, ILLINOIS 61801 AND RÉNYI INST. OF THE HUNGARIAN ACADEMY, 1364 \\ BUDAPEST, POB 127, HUNGARY \\ Radhika Ramamurthi† \\ DEPARTMENT OF MATHEMATICS, UNIVERSITY OF CALIFORNIA AT \\ SAN DIEGO, LA JOLLA, CA 92093
}

\begin{abstract}
The notion of a split coloring of a complete graph was introduced by Erdös and Gyárfás [7] as a generalization of split graphs. In this paper, we offer an alternate interpretation by comparing such a coloring to the classical Ramsey coloring problem via a two-round game played against an adversary. We show that the techniques used and bounds obtained on the extremal $(r, m)$-split coloring problem of [7] are closer in nature to the Turán theory of graphs rather than Ramsey theory. We extend the notion of these colorings to hypergraphs and provide bounds and some exact results. (c) (Year) John Wiley ES Sons, Inc.
\end{abstract}

Keywords: graphs, hypergraphs, Ramsey colorings, Turán's theorem, total colorings, group divisible designs

* Research supported in part by the Hungarian National Science Foundation grant OTKA 016389 and by NSF grant DMS-9970270.

$\dagger$ Research was done as a graduate student at the Department of Mathematics, University of Illinois, Urbana, Illinois 61801

Journal of Graph Theory Vol. (Volume Number), 1-11 ((Year))

(C) (Year) John Wiley \& Sons, Inc.

CCC (cccline information) 


\section{JOURNAL OF GRAPH THEORY}

\section{TOTAL COLORING GAMES}

Consider the following game played on an $n$-vertex complete graph against an adversary. We are given positive integers $r$ and $m$, and we have to start the game by coloring all the edges of the given clique with $r$ colors. The adversary then takes over and colors the vertices with $r$ colors, completing a total coloring. We lose, if at the end of the two rounds, the two colorings together contain a totally monochromatic $m$-clique. A totally monochromatic graph is one whose edges and vertices all have the same color.

If the edge-coloring we produce contains a monochromatic $m$-clique of color $i$, then the adversary will win by coloring these vertices with color $i$. So, our aim is to produce an edge-coloring that contains no monochromatic $m$-cliques. If the given $n$ is at least as large as the Ramsey number $R_{r}(m)$, then every edge-coloring contains a monochromatic $K_{m}$ in some color and the adversary will win. Below this threshold, there is always an edge-coloring that avoids monochromatic $m$-cliques. Such an edge-coloring is a Ramsey coloring, and these are the colorings that are favorable for us to win the "Total Ramsey Game".

Suppose, the adversary now changes the rules of the game and decides that we will win the game if there is a totally monochromatic $m$ - clique, instead of losing it. We ask how this changes the game and the threshold for winning.

Clearly, our aim now changes to producing an edge-coloring that has many monochromatic $m$-cliques in different colors. For the adversary to win, he must split (or destroy) these cliques by coloring some vertex with a different color. Let us call this game a "Total Split Game" and an edge-coloring of $K_{n}$ that is favorable to the adversary, an $(r, m)$-splittable coloring to indicate that the monochromatic $m$-cliques can all be split by an appropriate vertex-coloring.

\section{DEFINITIONS}

Formally, an edge-coloring of $K_{n}$ is called an $(r, m)$-splittable coloring if there is an $r$ vertex-coloring of $K_{n}$ that combines with the edge-coloring to avoid any totally monochromatic $K_{m}$. In other words, given an $r$-edge-coloring of $K_{n}$ which is $(r, m)$-splittable, the vertices can be partitioned into $r$ sets $V_{1}, \cdots, V_{r}$ so that for each $i$, the subclique induced by $V_{i}$ has no monochromatic $K_{m}$ in color $i$.

These colorings were introduced by Erdős and Gyárfás in [7] where they observed that for small complete graphs, every edge-coloring is a splittable coloring. It is easy to see that if we have an $r$-edge-coloring of $K_{n}$ that is not $(r, m)$-splittable, it can be extended to a non- $(r, m)$-splittable edge-coloring of $K_{n^{\prime}}$ for $n^{\prime}>n$. Therefore it is of interest to study the threshold where non-splittable colorings emerge. From the perspective of the Total Split Game, this is precisely the threshold beyond which we win against the adversary.

Let $f_{r}(m)$ be the minimum $n$ for which there is an $r$-edge-coloring of $K_{n}$ which is not $(r, m)$-splittable. 
(TITLE RUNNING HEAD) 3

Once $n$ is at least $f_{r}(m)$, there is an $r$-edge-coloring that wins the Total Split Game for us. One way to construct such an edge-coloring is to ensure that every set of size $\left\lceil\frac{n}{r}\right\rceil$ has monochromatic $m$-cliques in every color. This is clearly an edge-coloring which will defeat the adversary. These colorings were introduced by Erdős and Gyárfás [7] under the name $(r, m)$-balanced colorings.

Let $g_{r}(m)$ be the minimum $n$ for which there is an $r$-edge-coloring of $K_{n}$ which is $(r, m)$-balanced.

Every $(r, m)$-balanced coloring is a non- $(r, m)$-splittable coloring, hence we see that $f_{r}(m) \leq g_{r}(m)$. It is conjectured by Erdős and Gyárfás [7] that this inequality is strict.

\section{COMPARING $f_{r}(m)$ AND $R_{r}(m)$}

The general upper bound, $f_{r}(m) \leq R_{r}(m+1)$ is given in [7]. For small values of $m$ and $r, f_{r}(m)$ may be larger than $R_{r}(m)$. For example, when $m=2, R_{r}(2)=2$ while in [7], Erdős and Gyárfás showed that $\left(\begin{array}{l}r \\ 2\end{array}\right)<f_{r}(2) \leq r^{2}+r+1$ (where the upper bound holds if there is a finite projective plane of order $r+1$ ).

In the following section, however, we will show that the order of magnitude of $f_{r}(m)$ is much smaller - of the form $O\left(r^{2} m^{2}\right)$, while $R_{r}(m)$ is known to be exponential. In fact, $R_{r}(m)$ exceeds $f_{r}(m)$ once $m \geq 5$. Further, the non-splittable coloring presented is an explicit construction while all known near-optimal Ramsey colorings are obtained using random methods.

\section{CONSTRUCTION FROM TRANSVERSAL DESIGNS}

To establish the upper bound, we construct a coloring with the use of combinatorial designs known as nets (see e.g. [4, p.97]).

Definition. A $(k, v)$-net is a pair $(X, C)$ where $X$ is a set of $v^{2}$ elements and $C$ is a set of $k v$ blocks, each of size $v$, with the property that two distinct blocks intersect in at most one element. Moreover, the blocks can be partitioned into $k$ parallel classes, each containing $v$ blocks.

Interchanging the roles of elements and blocks gives us a $(v, k)$-transversal design.

Theorem 1. $f_{r}(m) \leq g_{r}(m) \leq v^{2}$ if there is an $(r, v)$-net for $v>r(m-1)$.

Proof. Let $n=v^{2}$. We will show that $K_{n}$ has an $(r, m)$-balanced coloring. Let $(X, C)$ be the $(r, v)$-net with the blocks in $C$ partitioned into $r$ parallel classes. Let $V\left(K_{n}\right)=X$ and color the edges as follows:

For $c \in[r]$, the color of edge $(i, j)$ is $c$ if and only if $i$ and $j$ lie together in some block in parallel class $c$. This colors only $r v\left(\begin{array}{l}v \\ 2\end{array}\right)$ of the edges in $K_{n}$. To color the remaining edges, we chose a color for each arbitrarily from $\{1,2, \ldots, r\}$. 
Now, let $S \subseteq X$ such that $|S| \geq\left\lceil\frac{n}{r}\right\rceil>v(m-1)$. Consider color class $c$. Since each class contains $v$ parallel blocks, some block must contain at least $m$ points from $S$, thus yielding a $K_{m}$ in color $i$.

A $(k, v)$-net can have at most $v+1$ parallel classes. To see this, observe that the total number of pairs of points is $\left(\begin{array}{c}v^{2} \\ 2\end{array}\right)$. Since each pair of points appears together in at most

one block, $k v\left(\begin{array}{l}v \\ 2\end{array}\right) \leq\left(\begin{array}{c}v^{2} \\ 2\end{array}\right)$. This yields $k \leq v+1$. When $k=v+1$, each pair appears exactly once, and a $(v+1, v)$-net is an affine plane. It is well known (see e.g., [4, p.76]) that an affine plane of order $v$ exists whenever $v$ is a prime power. Thus we obtain,

Corollary 2. $f_{r}(m) \leq g_{r}(m) \leq v^{2}$ where $v$ is a power of a prime such that $v>r(m-$ $1)$.

In general, the existence of $(k, v)$-nets was shown to be equivalent to the existence of $(k-2)$ mutually orthogonal Latin Squares of side $v$ by Bose (see e.g. [12, p.253]). Chowla, Erdős and Straus [6] proved that for fixed $k$ and sufficiently large $v$, there is a $(k, v)$-net. So, we obtain the following:

Corollary 3. For fixed $r$ and $m$ sufficiently large, $f_{r}(m) \leq g_{r}(m) \leq(r(m-1)+1)^{2}$.

\section{LOWER BOUNDS FROM EXTREMAL GRAPH THEORY}

We present two lower bounds for $f_{r}(m)$, extending the ideas given by Erdős and Gyárfás in $[7]$.

Theorem 4. $f_{r}(m) \geq 2(r-1)\left(\begin{array}{c}m \\ 2\end{array}\right)+m$.

Proof. Let $n=(r-1) m(m-1)+(m-1)$ and consider any $r$-edge-coloring of $K_{n}$. Select the maximum number of vertex disjoint monochromatic $m$-cliques of color 1 and color the remaining vertices with color 1 . (There are at least $(m-1)$ such vertices.) Now, divide the monochromatic $m$-cliques of color 1 into $(r-1)$ classes, each containing at most $(m-1)$ copies. This is possible since the number of vertices remaining is at most $(r-1) m(m-1)$. Then each class can be colored with a different color from $\{2, \ldots, r\}$ and no totally monochromatic $K_{m}$ is formed.

We remark here that this proof can be modified to obtain a slightly better lower bound on $g_{r}(m)$ :

$$
g_{r}(m) \geq r m(m-1)+1 .
$$

We can now conclude from Corollary 3 and Theorem 4 that

$$
r \leq \liminf _{m \rightarrow \infty} \frac{f_{r}(m)}{m^{2}} \leq \limsup _{m \rightarrow \infty} \frac{g_{r}(m)}{m^{2}} \leq r^{2} .
$$


(TITLE RUNNING HEAD) 5

Theorem 5. For $r \geq m \geq 3, f_{r}(m) \geq(m-1)\left(\left(\begin{array}{c}r \\ 2\end{array}\right)+\left(\begin{array}{c}m \\ 2\end{array}\right)\right)+(m-3)(r-m)+m$.

For the proof we use the following lemma due to Alon, Kahn and Seymour, [2, Corollary 1.4] and which is, in fact, a generalization of the classical Turán's theorem [11].

Lemma 1. If $e(G) \leq \frac{1}{r}\left(\begin{array}{c}n(G) \\ 2\end{array}\right)$ where $n \geq(m-1)\left(\begin{array}{l}r \\ 2\end{array}\right)$, then there is an $S \subseteq V(G)$, $|S| \geq(m-1) r-2$ and $\omega\left(\left.G\right|_{S}\right)<m$, for $r \geq 5$.

The proof of Theorem 5 now follows by induction.

Proof. We prove the theorem by induction on $r$. The base case is Theorem 4 with $r=m \geq 5$. So, assume $r>m$ and consider an arbitrary $r$-edge-coloring of $K_{n}$, where $n=(m-1)\left(\left(\begin{array}{c}r \\ 2\end{array}\right)+\left(\begin{array}{c}m \\ 2\end{array}\right)\right)+(m-3)(r-m)+m-1$. By the lemma, there is a color class, say color 1 , with a subset of the vertices, $S$ such that $S$ contains no $K_{m}$ in color 1 and $|S| \geq r(m-1)-2$.

Delete $S$ from $V\left(K_{n}\right)$. We now have at most $(m-1)\left(\left(\begin{array}{c}r-1 \\ 2\end{array}\right)+\left(\begin{array}{c}m \\ 2\end{array}\right)\right)+(m-3)(r-$ $1-m)+m-1$ vertices remaining. By changing color 1 to color 2 in this clique, we can apply the induction hypothesis to partition the vertices into $S_{2}, S_{3}, \cdots, S_{r}$ such that $S_{i}$ has no $K_{m}$ of color $i$. Adding $S=S_{1}$, we obtain the desired partition for $K_{n}$.

Using density results for prime powers (see e.g. [5, p.154]), Corollary 2 and Theorem 5 now give us the following :

$$
m-1 \leq \liminf _{r \rightarrow \infty} \frac{f_{r}(m)}{r^{2}} \leq \limsup _{r \rightarrow \infty} \frac{g_{r}(m)}{r^{2}} \leq m^{2} .
$$

\section{GENERALIZATION TO HYPERGRAPHS}

Let $\mathcal{K}_{n}^{k}$ denote the complete $k$-uniform hypergraph on $n$ vertices, i.e. we have a ground set of $n$ elements and we take all the $k$-sets to be edges. Often, we will denote the ground set $\{1,2, \cdots, n\}$ by $[n]$. An $r$-edge-coloring of $\mathcal{K}_{n}^{k}$ is a labeling of the $k$-sets with $\{1,2, \ldots, r\}$. Given a coloring of the vertices and edges of $\mathcal{K}_{n}^{k}$, a totally monochromatic $m$-clique, for $k \leq m \leq n$ is a $\mathcal{K}_{m}^{k}$, whose vertices and edges ( $k$-sets) all get the same color.

Clearly, the games that were defined in Section 1. can played on hypergraphs, instead of graphs. The threshold value of $n$ above which the adversary wins for the Total Ramsey Game is again the classical hypergraph Ramsey number $R_{r}^{k}(m)$.

In the following sections, we investigate the Total Split Game on hypergraphs. We first extend the previous definitions to hypergraphs.

Definition. An $r$-coloring of $\mathcal{E}\left(\mathcal{K}_{n}^{k}\right)$ is $(r, m)$-splittable if there is a coloring of $V\left(\mathcal{K}_{n}^{k}\right)$ with $r$ colors so that no totally monochromatic $m$-clique is produced.

Let $f_{r}^{k}(m)$ be the minimum $n$ for which there is an $r$-coloring of $\mathcal{E}\left(\mathcal{K}_{n}^{k}\right)$ that is not $(r, m)$-splittable.

If we give the adversary a hypergraph whose edges have been colored with an $(r, m)$ splittable coloring, the adversary can color the vertices without producing any totally 
monochromatic $m$-cliques and win the Total Split Game. Once the number of vertices in the hypergraph is at least $f_{r}^{k}(m)$, however, we can find an $r$-coloring of the $k$-sets of $\mathcal{K}_{n}^{k}$ with the property that every coloring of the vertices with $r$ colors produces a totally monochromatic $m$-clique. This defeats the adversary, so $f_{r}^{k}(m)$ is the threshold value of $n$ beyond which we win the Total Split Game on $\mathcal{K}_{n}^{k}$. Our results in the next section suggest that when $r$ and $m$ are fixed, this value decreases as $k$ increases. To investigate the threshold, we extend the definition of balanced colorings to hypergraphs.

Definition. An $r$-coloring of $\mathcal{E}\left(\mathcal{K}_{n}^{k}\right)$ where every set of size $\left\lceil\frac{n}{r}\right\rceil$ has a monochromatic $m$-clique in every color is an $(r, m)$-balanced coloring. Let $g_{r}^{k}(m)$ be the minimum $n$ for which there is an $r$-coloring of $\mathcal{E}\left(\mathcal{K}_{n}^{k}\right)$ that is $(r, m)$-balanced.

As before, an $(r, m)$-balanced coloring is not $(r, m)$-splittable, and hence $f_{r}^{k}(m) \leq$ $g_{r}^{k}(m)$

\section{LOWER BOUNDS FOR HYPERGRAPHS}

We first observe that the same argument as in Theorem 4 gives:

Theorem 6. $f_{r}^{k}(m) \geq m(r-1)\left\lfloor\frac{m-1}{k-1}\right\rfloor+m$.

Proof. Let $n \leq m(r-1)\left\lfloor\frac{m-1}{k-1}\right\rfloor+(m-1)$ and consider any $r$-coloring of $\mathcal{E}\left(\mathcal{K}_{n}^{k}\right)$. We exhibit a coloring of the vertices to show that the edge-coloring is $(r, m)$-splittable.

Select the maximum number of vertex-disjoint copies of $\mathcal{K}_{m}^{k}$ so that in each copy of all the edges are of color 1 . Color the remaining vertices with color 1 (there are at least $(m-1)$ such vertices). Now, partition the disjoint copies of $\mathcal{K}_{m}^{k}$ whose edges all have color 1 into $(r-1)$ classes $C_{2}, C_{3} \ldots, C_{r}$, each containing at most $\frac{m-1}{k-1}$ of these copies. Color the vertices in $U_{i}$ with color $i$. In forming a totally monochromatic $\mathcal{K}_{m}^{k}$ in color $i$ for $i>1$, at most $k-1$ vertices can be taken from any of the copies of $\mathcal{K}_{m}^{k}$ that are edge-monochromatic in color 1 . Hence from $U_{i}$ we can pick at most $m-1$ vertices all of whose $k$-sets have color $i$, and no totally monochromatic copy of $\mathcal{K}_{m}^{k}$ is formed.

A slight modification of the above argument, as remarked before, also gives us:

Theorem 7. $g_{r}^{k}(m)>r m\left\lfloor\frac{m-1}{k-1}\right\rfloor$.

We do not have a hypergraph extension of Theorem 5. For the special case $m=k$, however, we use a result of Alon's [1] on hypergraphs with high chromatic number to prove a lower bound on $f_{r}^{k}(k)$. When the number of edges in a $k$-uniform hypergraph is at most $((k-1) s / k)^{k},[1$, Proposition 2] guarantees a stable set of size at least $n / s$. Applying this to the hypergraph obtained by considering the edges of the least frequent color in any $r$-edge-coloring of $\mathcal{K}_{n}^{k}$ for $k \geq 3$, we obtain a stable set of size $k r^{1 / k} / 3$. For $r \geq k$, induction on $r$ gives a lower bound on $f_{r}^{k}(k)$ of $\frac{k^{2}}{3(k+1)}\left(r^{1+1 / k}-k^{1+1 / k}\right)+k^{2}$. 
(TITLE RUNNING HEAD) 7

\section{ALGEBRAIC CONSTRUCTION FOR UPPER BOUND}

We provide an upper bound for $f_{r}^{k}(m)$ by constructing an $(r, m)$-balanced coloring.

Theorem 8. $f_{r}^{k}(m) \leq g_{r}^{k}(m) \leq \frac{q^{2}-1}{t}$ where $q \equiv 1 \bmod \mathrm{t}$ is a power of a prime such that $q \geq r(m+1)-1$ and $t<k$.

Proof. To obtain the coloring, we consider an algebraic construction defined in [8].

Let $\mathbb{F}_{q}$ be the $q$-element finite field. Let $\theta$ be a primitive element; $\theta$ is a generator of the multiplicative group of nonzero elements of $\mathbb{F}_{q}$. Since $q \equiv 1 \bmod \mathrm{t}$, we have that $h=\theta^{(q-1) / t}$ is an element of order $t$, and $H=\left\{1, h, h^{2}, \cdots, h^{t-1}\right\}$ forms a $t$-element subgroup of $\mathbb{F}_{q}-\{0\}$.

We define a relation $\sim$ on $\left(\mathbb{F}_{q} \times \mathbb{F}_{q}\right)-\{(0,0)\}$. Let $(a, b) \sim\left(a^{\prime}, b^{\prime}\right)$ if there exists some $h^{\alpha}$ such that $a^{\prime}=h^{\alpha} a$ and $b^{\prime}=h^{\alpha} b$. It is immediate that $\sim$ is an equivalence relation. Each equivalence class consists of $t$ pairs $(a, b)$ from $\left(\mathbb{F}_{q} \times \mathbb{F}_{q}\right)-\{(0,0)\}$. Let $\langle a, b\rangle$ denote the class containing $(a, b)$. There are $\frac{q^{2}-1}{t}$ equivalence classes, and these will form the vertex set $V$ of our complete $k$-uniform hypergraph.

Define $L(\langle a, b\rangle)=\{\langle x, y\rangle: a x+b y \in H\}$. Given $(a, b)$ with $b \neq 0$, for any given $x$ and $h^{\alpha}$ the equation $a x+b y=h^{\alpha}$ has a unique solution for $y$. Thus, there are $t q$ solutions $(x, y)$, but these come in equivalence classes, so $|L(\langle a, b\rangle)|=q$.

We view $L(\langle a, b\rangle)$ as a line passing through $q$ points of $V$. Füredi [8] showed that every two lines intersect in at most $t$ points. For each line $L(\langle a, b\rangle)$, the set $\left\{L\left(\left\langle\theta^{i} a, \theta^{i} b\right\rangle\right)\right.$ : $\left.1 \leq i<\frac{q-1}{t}\right\}$ is a set of pairwise disjoint lines containing $L(\langle a, b\rangle)$. We call such a set of pairwise disjoint lines a parallel class. Since each line covers $q$ elements of $V$, each parallel class covers all but $\frac{q-1}{t}$ elements of $V$.

From the set of $\frac{q^{2}-1}{t}$ lines, we can form $q+1$ parallel classes $P_{1}, \ldots, P_{q+1}$ and use these to define an $r$-coloring of the $k$-sets in $V$. A $k$-set receives color $i$ if it is contained in some $L(\langle a, b\rangle)$ in class $P_{i}$, for $i \leq i \leq r$. The remaining $k$-sets are colored arbitrarily. This is well-defined, since two lines intersect in at most $t$ points, and we have $t<k$ by hypothesis.

We claim that this coloring is $(r, m)$-balanced. Take $S \subseteq V$ such that $|S| \geq\left\lceil\frac{n}{r}\right\rceil \geq$ $\frac{q^{2}-1}{t r}$. Consider color class $i$. Since each parallel class omits $\frac{q-1}{t}$ elements of $V$, the lines in class $P_{i}$ together contain at least $\frac{q^{2}-1}{t r}-\frac{q-1}{t}$ points of $S$. Since each class contains at most $\frac{q-1}{t}$ parallel lines, some line must contain at least $\left(\frac{q^{2}-1}{t r}-\frac{q-1}{t}\right) \frac{t}{q-1}$ points from $S$. Since $\left(\frac{q^{2}-1}{t r}-\frac{q-1}{t}\right) \frac{t}{q-1}=\frac{q+1}{r}-1 \geq m$, this yields an $m$-clique in color $i$.

It is a well-known result in number theory that for fixed $t$ and every $x \in \mathbb{N}$, there is a prime number congruent to 1 mod t between $x$ and $x+o(x)$, (see e.g. [5], page 154). If we set $t=k-1$ and let $r$ and $k$ be fixed while $m$ grows, then this number-theoretic result together with Theorems 6 and 8 yields

$$
\frac{r}{k-1} \leq \liminf _{m \rightarrow \infty} \frac{f_{r}^{k}(m)}{m^{2}} \leq \limsup _{m \rightarrow \infty} \frac{g_{r}^{k}(m)}{m^{2}} \leq \frac{r^{2}}{k-1} .
$$


JOURNAL OF GRAPH THEORY

\section{SOME EXACT RESULTS WHEN $k=m$}

Theorem 9. $f_{2}^{k}(k)=2 k$ for $k \neq 5,7$.

Proof. From Theorem 6, we get that $f_{2}^{k}(k) \geq 2 k$.

We need to exhibit a 2-coloring of the $k$-sets of $[2 k]$ such that every 2-coloring of the vertices creates a totally monochromatic edge. We note that such an edge-coloring can be equivalently described as having the following properties:

(i) every $k$-set and its complement get the same color,

(ii) every $(k+1)$-set contains a $k$-set of each color.

For even $k$, we can explicitly describe this coloring of the $k$-sets as follows: fix a $k$-set, $A$. The color of the $k$-set, $F$ is $|F \cap A| \bmod 2$. Since $|F \cap A|+|\bar{F} \cap A|=|A|=k$, which is even, property (i) is ensured. If $B$ is any $(k+1)$-set, then $B \subset[2 k]$ implies that there is an $x \in A \cap B$ and a $y \in \bar{A} \cap B$. Then, $B-x$ and $B-y$ are $k$-sets of $B$ with different colors and we get property (ii).

For $k=3$, a non- $(2, k)$-splittable coloring can be obtained by giving the triples $123,124,135,146,156$ and their complements color 0 and all remaining triples color 1 . A little case analysis shows that property (ii) also holds, and the result follows.

For $k \geq 9$, we will use the Lovász Local Lemma, (see e.g.[3, p.53]), to show that a suitable random coloring has properties (i) and (ii). Consider all the $k$-sets of $[2 k]$ that contain the element 1 and color them with 2 colors randomly and independently. Extend this to a coloring of all $k$-sets by giving any uncolored $k$-set the color of its complementary $k$-set. This ensures that (i) holds.

For each $(k+1)$-set, $B$, let $F_{B}$ be the event that all $k$-sets of $B$ get the same color. Then, $\operatorname{Pr}\left(F_{B}\right)=2^{-k} . F_{B}$ is mutually independent of all events $F_{B^{\prime}}$, such that $2<\left|B \cap B^{\prime}\right|<k$. Thus, $F_{B}$ is mutually independent of all but at most $(k+1)(k-1)+\left(\begin{array}{c}k+1 \\ 2\end{array}\right)$ events. By the Local Lemma, a 2-coloring with no $(k+1)$-set having all $k$-sets of the same color exists if $\frac{k(3 k+1)}{2} 2^{-k} e<1$, which is true if $k \geq 9$.

Theorem 10. $g_{r}^{k}(k)=r k+1$ for $r \leq \frac{k+1}{4 \ln (k+1)}$.

Proof. From Theorem 7, we know that $g_{r}^{k}(k) \geq r k+1$. We need to exhibit an $r$ coloring of the $k$-sets of $[r k+1]$ with the property that every $(k+1)$-set contains a $k$-set of each color.

Consider a coloring where each $k$-set from $[r k+1]$ is colored with one of the $r$ available colors randomly and independently. For each $(k+1)$-set, $B$, let $F_{B}$ be the event that not all colors are present on the $k$-sets of $B$. Then, $\operatorname{Pr}\left(F_{B}\right) \leq r\left(1-\frac{1}{r}\right)^{k+1} \cdot F_{B}$ is mutually independent of all events $F_{B^{\prime}}$, unless $\left|B \cap B^{\prime}\right|=k$. Thus there are at most $(k+1) k(r-1)$ events of which $F_{B}$ is not independent. Applying the Local Lemma again, we find that a balanced $r$-coloring exists if $r \leq \frac{k+1}{4 \ln (k+1)}$.

In order to have an $(r, k)$-balanced coloring, every set of order $\lceil n / r\rceil$ must contain at least $r$ edges. This implies that if $n=r k+1$, then $r \leq k+1$. So, it remains to be determined if $g_{r}^{k}(k)$ is also $r k+1$ for values of $r$ between $\frac{k+1}{4 \ln (k+1)}$ and $k+1$. 
(TITLE RUNNING HEAD) 9

Recall that $f_{r}^{k}(k) \leq g_{r}^{k}(k)$ always. We now show that $r k+1$ is also a lower bound for $f_{r}^{k}(k)$ whenever $r$ is at least 3 .

Theorem 11. $f_{r}^{k}(k) \geq r k+1$ for $r \geq 3$.

Proof. Take any $r$-coloring of $\mathcal{E}\left(\mathcal{K}_{n}^{k}\right)$ where $n=r k$. We give an $r$ coloring of the vertices so that no totally monochromatic $k$-set is formed. Since the number of vertices equals the number of colors times the edge size, it suffices to assign each color only to vertices of an edge that received a different color. This avoids creating a totally monochromatic $k$-clique.

Choose a $k$-set and assign its vertices a color different from the color assigned to it by the edge-coloring. Eliminate this color. Continue choosing disjoint $k$-sets and coloring their vertices in this way until all but 3 colors from $[r]$ are used. Let 1,2,3 denote the colors remaining, and let $V$ denote the set of $3 k$ vertices remaining.

If some $k$-set $A$ in $V$ is not colored from $\{1,2,3\}$, then we first color the remaining $2 k$ vertices by picking some two disjoint $k$-sets and giving their vertices colors from $\{1,2,3\}$ so that the vertices do not get the same color as the $k$-set. The third color can be assigned to the vertices of $A$. So, we may assume that every $k$-set in $V$ is colored from $\{1,2,3\}$. If there are two disjoint $k$-sets with different colors, then we can assign the colors from $\{1,2,3\}$ to the vertices of $k$-sets so that the color on no $k$-set is the same as the color on its vertices. So, we may assume that every pair of disjoint $k$-sets in $V$ has the same color. Let $A$ be a $k$-set in $V$, we may assume that it has color 1 . All the $k$-sets in $V-A$ also have color 1 . So, give all the vertices of $A$ color 2 and all the vertices of $V-A$ color 3 . Note that in this final case we did not use all the colors on vertices.

Theorems 10 and 11 immediately imply

Corollary 12. $f_{r}^{k}(k)=r k+1$ for $3 \leq r \leq \frac{k+1}{4 \ln (k+1)}$.

\section{FURTHER PROBLEMS - SPLITTABLE COLORINGS BEYOND CLIQUES}

The Total Split Game can be generalized by requiring the adversary to split graphs other than cliques.

Let $\mathcal{F}=\left(F_{1}, F_{2}, \ldots, F_{r}\right)$ be a list of graphs. The Total Split Game $T S(r, \mathcal{F}, n)$ is defined as follows. In the first round, we color the edges of $K_{n}$ with $r$ colors. In the second round the adversary colors the vertices with $r$ colors, completing a total coloring. We win if this total coloring contains a totally monochromatic copy of $F_{i}$ in color $i$, for some $i$. If $\mathcal{F}=\left(K_{m}, \ldots, K_{m}\right)$, then this is the same as the Total Split Game $T S(r, m, n)$ discussed earlier.

An $r$-edge-coloring favors the adversary if there is some $r$-vertex-coloring that splits every copy of $F_{i}$ whose edges all have color $i$. 
Definition. Given a list of graphs $\mathcal{F}=\left(F_{1}, F_{2}, \cdots, F_{r}\right)$ and an $n$-vertex clique $K_{n}$, an $r$-coloring of the edges of $K_{n}$ is called $\mathcal{F}$-splittable if there is a partition of the vertex set of $K_{n}$ into $V_{1}, \ldots, V_{r}$ such that the graph induced by $V_{i}$ has no monochromatic copy of $F_{i}$ in color $i$, for each $i$. Let $f_{r}(\mathcal{F})$ be the minimum $n$ for which there is an $r$-edge-coloring of $K_{n}$ that is not $\mathcal{F}$-splittable.

Let $\mathcal{K}(\mathcal{F})$ denote the family obtained by replacing each graph in $\mathcal{F}$ by a clique of the same order, then clearly, $f_{r}(\mathcal{F})<f_{r}(\mathcal{K}(\mathcal{F}))$. Similarly, if $\mathcal{K}(\omega(\mathcal{F}))$ is the family obtained by replacing each graph in $\mathcal{F}$ by the largest clique it contains as a subgraph, we see that $f_{r}(\mathcal{F}) \geq f_{r}(\mathcal{K}(\omega(\mathcal{F})))$.

The Total Split Game also generalizes naturally to edge-colorings of arbitrary graphs. Since an arbitrary graph with $n$ vertices has fewer edges to color than $K_{n}$, it is easier to split all copies of $F_{i}$ in each color $i$, since there tend to be fewer of these copies. The definition of $\mathcal{F}$-splittable for edge-colorings of a graph $G$ is as for $G=K_{n}$ given above. By analogy with $f_{r}(\mathcal{F})$, when $\mathcal{G}$ is a family of graphs we introduce $f(\mathcal{G} ; \mathcal{F})$ to mean the minimum of $n(G)$ such that $G \in \mathcal{G}$ and $G$ has a non-splittable coloring. Note that $\mathcal{G}$ and $\mathcal{F}$ must be chosen appropriately for the parameter to exist.

So, similar to the generalizations of Ramsey and Turán theory from cliques to other graphs, the above ideas of splittable colorings pose a series of new extremal problems.

\section{ACKNOWLEDGMENTS}

The authors are grateful to André Kündgen for valuable discussions and for suggestions on the manuscript. Thanks also to Eric Mendelsohn on suggesting the use of nets to extend Theorem 1 and to Douglas West for his suggestions. This research was completed while the second author visited the Fields Institute for Research in Mathematical Sciences, Toronto and its hospitality is greatly appreciated.

\section{References}

[1] Alon,N., "Hypergraphs with high chromatic number", Graphs and Combinatorics 1, 1985, pp. 387-389.

[2] Alon,N., Kahn,J. and Seymour,P.D., "Large induced degenerate subgraphs", Graphs and Combinatorics 3, 1987, pp. 203-211.

[3] Alon,N., Spencer,J., "The Probabilistic Method", Wiley-Interscience Series in Discrete Math and Optimization, 1992.

[4] Anderson,I., "Combinatorial Designs and Tournaments", Oxford Science Publications, 1997.

[5] Apostol,T., "Introduction to Analytic Number Theory", 2nd edition, Springer-Verlag, 1986.

[6] Chowla,S., Erdős,P., Straus,E.G., "On the maximal number of pairwise orthogonal Latin squares of a given order", Canadian Journal of Mathematics 13, 1960, pp. 204-208 
(TITLE RUNNING HEAD) 11

[7] Erdős,P. and Gyárfás,A., "Split and balanced colorings of complete graphs", Discrete Mathematics 200, 1999, pp. 79-86.

[8] Füredi,Z., "New Asymptotics for Bipartite Turán Numbers", Journal of Combinatorial Theory, Series A 75, 1996, pp. 141-144.

[9] Gyárfás,A., "Generalized split graphs and Ramsey numbers", Journal of Combinatorial Theory, Series A 81, 1998, pp. 255-261.

[10] Gyárfás,A., Kézdy,A. and Lehel,J., "Split Colorings", to appear in Discrete Mathematics.

[11] Turán,P., "On an extremal problem in graph theory" (in Hungarian), Mat. Fiz. Lapok, 48, 1941, pp. 436-452.

[12] van Lint,J. H., Wilson,R. M., "A course in combinatorics", Cambridge University Press, Cambridge, 1992. 